\title{
A DARK ENERGY CAMERA SEARCH FOR AN OPTICAL COUNTERPART TO THE FIRST ADVANCED LIGO GRAVITATIONAL WAVE EVENT GW150914
}

M. Soares-Santos ${ }^{1}$, R. Kessler ${ }^{2}$, E. Berger ${ }^{3}$, J. Annis ${ }^{1}$, D. Brout ${ }^{4}$, E. Buckley-Geer ${ }^{1}$, H. Chen ${ }^{2}$, P. S. Cowperthwaite ${ }^{3}$, H. T. Diehl ${ }^{1}$, Z. Doctor ${ }^{2}$, A. Drlica-Wagner ${ }^{1}$, B. Farr $^{2}$, D. A. Finley ${ }^{1}$, B. Flaugher ${ }^{1}$, R. J. Foley ${ }^{5,6}$, J. Frieman ${ }^{1,2}$,

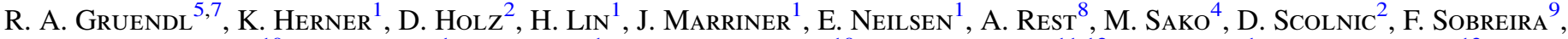
A. R. Walker ${ }^{10}$, W. Wester ${ }^{1}$, B. Yanny ${ }^{1}$, T. M. C. Abbott ${ }^{10}$, F. B. Abdalla ${ }^{11,12}$, S. Allam ${ }^{1}$, R. Armstrong ${ }^{13}$,

M. Banerji ${ }^{14,15}$, A. Benoit-Lévy ${ }^{11,16,17}$, R. A. Bernstein ${ }^{18}$, E. Bertin ${ }^{16,17}$, D. A. Brown ${ }^{19}$, D. L. Burke ${ }^{20,21}$, D. CAPOZZI ${ }^{22}$, A. Carnero Rosell ${ }^{23,24}$, M. Carrasco Kind ${ }^{5,7}$, J. Carretero ${ }^{25,26}$, F. J. Castander ${ }^{25}$, S. B. Cenko ${ }^{27,28}$, R. Chornock ${ }^{29}$, M. Crocce ${ }^{25}$, C. B. D'ANdrea ${ }^{22,30}$, L. N. DA Costa ${ }^{23,24}$, S. Desai $^{31,32}$, J. P. Dietrich ${ }^{31,32}$, M. R. Drout ${ }^{3}$, T. F. EIFLER ${ }^{4,33}$, J. Estrada ${ }^{1}$, A. E. Evrard ${ }^{34,35}$, S. Fairhurst ${ }^{36}$, E. Fernandez ${ }^{26}$, J. Fischer ${ }^{4}$, W. FonG ${ }^{37}$, P. Fosalba ${ }^{25}$, D. B. Fox ${ }^{38}$, C. L. Fryer ${ }^{39}$, J. Garcia-Bellido ${ }^{40}$, E. Gaztanaga ${ }^{25}$, D. W. Gerdes ${ }^{35}$, D. A. Golddstein ${ }^{41,42}$, D. Gruen ${ }^{20,21}$, G. Gutierrez ${ }^{1}$,

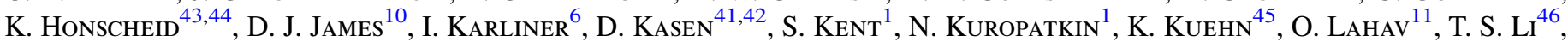
M. Lima $^{23,47}$, M. A. G. Maia ${ }^{23,24}$, R. Margutti ${ }^{48}$, P. Martini ${ }^{43,49}$, T. Matheson ${ }^{50}$, R. G. McMahon ${ }^{14,15}$, B. D. MetzGer ${ }^{51}$, C. J. Miller ${ }^{34,35}$, R. MiQuel ${ }^{26,52}$, J. J. Mohr ${ }^{31,32,53}$, R. C. Nichol ${ }^{22}$, B. Nord ${ }^{1}$, R. Ogando ${ }^{23,24}$, J. Peoples ${ }^{1}$, A. A. Plazas ${ }^{33}$, E. QuataerT ${ }^{54}$, A. K. Romer ${ }^{55}$, A. Roodman ${ }^{20,21}$, E. S. RykofF ${ }^{20,21}$, E. SAnchez ${ }^{40}$, V. Scarpine ${ }^{1}$, R. SchindleR ${ }^{21}$, M. Schubnell ${ }^{35}$, I. Sevilla-Noarbe ${ }^{5,40}$, E. Sheldon ${ }^{56}$, M. Smith ${ }^{30}$, N. Smith $^{57}$, R. C. Smith ${ }^{10}$, A. Stebbins ${ }^{1}$, P. J. Sutton ${ }^{58}$, M. E. C. Swanson ${ }^{7}$, G. Tarle ${ }^{35}$, J. Thaler ${ }^{6}$, R. C. Thomas ${ }^{42}$, D. L. Tucker ${ }^{1}$, V. Vikram ${ }^{59}$, R. H. WeCHSLER ${ }^{20,21,60}$, AND J. WELLER ${ }^{31,53,61}$

(The DES Collaboration)

${ }^{1}$ Fermi National Accelerator Laboratory, P.O. Box 500, Batavia, IL 60510, USA

${ }^{2}$ Kavli Institute for Cosmological Physics, University of Chicago, Chicago, IL 60637, USA

${ }^{3}$ Harvard-Smithsonian Center for Astrophysics, 60 Garden Street, Cambridge, MA 02138, USA

${ }^{4}$ Department of Physics and Astronomy, University of Pennsylvania, Philadelphia, PA 19104, USA

${ }^{5}$ Department of Astronomy, University of Illinois, 1002 W. Green Street, Urbana, IL 61801, USA

${ }^{6}$ Department of Physics, University of Illinois, 1110 W. Green Street, Urbana, IL 61801, USA

${ }^{7}$ National Center for Supercomputing Applications, 1205 West Clark Street, Urbana, IL 61801, USA ${ }^{8}$ STScI, 3700 San Martin Dr., Baltimore, MD 21218, USA

${ }^{9}$ Instituto de Física Teórica, Universidade Estadual Paulista, Rua Dr. Bento T. Ferraz 271, São Paulo, SP 01140-070, Brazil

${ }^{10}$ Cerro Tololo Inter-American Observatory, National Optical Astronomy Observatory, Casilla 603, La Serena, Chile

${ }_{11}^{11}$ Department of Physics \& Astronomy, University College London, Gower Street, London, WC1E 6BT, UK

${ }^{12}$ Department of Physics and Electronics, Rhodes University, P.O. Box 94, Grahamstown, 6140, South Africa

${ }^{13}$ Department of Astrophysical Sciences, Princeton University, Peyton Hall, Princeton, NJ 08544, USA

${ }^{14}$ Institute of Astronomy, University of Cambridge, Madingley Road, Cambridge CB3 OHA, UK

${ }^{15}$ Kavli Institute for Cosmology, University of Cambridge, Madingley Road, Cambridge CB3 OHA, UK

${ }^{16}$ CNRS, UMR 7095, Institut d'Astrophysique de Paris, F-75014, Paris, France
${ }^{17}$ Sorbonne Universités, UPMC Univ Paris 06, UMR 7095, Institut d'Astrophysique de Paris, F-75014, Paris, France

${ }^{18}$ Carnegie Observatories, 813 Santa Barbara St., Pasadena, CA 91101, USA

${ }^{19}$ Physics Department, Syracuse University, Syracuse, NY 13244, USA

${ }^{20}$ Kavli Institute for Particle Astrophysics \& Cosmology, P.O. Box 2450, Stanford University, Stanford, CA 94305, USA

${ }^{21}$ SLAC National Accelerator Laboratory, Menlo Park, CA 94025, USA

${ }^{22}$ Institute of Cosmology \& Gravitation, University of Portsmouth, Portsmouth, PO1 3FX, UK

${ }^{23}$ Laboratório Interinstitucional de e-Astronomia-LIneA, Rua Gal. José Cristino 77, Rio de Janeiro, RJ-20921-400, Brazil

${ }^{24}$ Observatório Nacional, Rua Gal. José Cristino 77, Rio de Janeiro, RJ-20921-400, Brazil

${ }^{25}$ Institut de Ciències de l'Espai, IEEC-CSIC, Campus UAB, Carrer de Can Magrans, s/n, E-08193 Bellaterra, Barcelona, Spain

${ }^{26}$ Institut de Física d'Altes Energies (IFAE), The Barcelona Institute of Science and Technology, Campus UAB, 08193 Bellaterra (Barcelona) Spain

${ }^{27}$ Astrophysics Science Division, NASA Goddard Space Flight Center, Mail Code 661, Greenbelt, MD 20771, USA

${ }^{28}$ Joint Space-Science Institute, University of Maryland, College Park, MD 20742, USA

${ }^{29}$ Astrophysical Institute, Department of Physics and Astronomy, 251B Clippinger Lab, Ohio University, Athens, OH 45701, USA

${ }^{30}$ School of Physics and Astronomy, University of Southampton, Southampton, SO17 1BJ, UK

${ }^{31}$ Excellence Cluster universe, Boltzmannstr. 2, D-85748 Garching, Germany

${ }^{32}$ Faculty of Physics, Ludwig-Maximilians-Universität, Scheinerstr. 1, D-81679 Munich, Germany
${ }^{33}$ Jet Propulsion Laboratory, California Institute of Technology, 4800 Oak Grove Drive, Pasadena, CA 91109, USA

${ }^{34}$ Department of Astronomy, University of Michigan, Ann Arbor, MI 48109, USA

${ }^{35}$ Department of Physics, University of Michigan, Ann Arbor, MI 48109, USA

${ }^{36}$ School of Physics and Astronomy, Cardiff University, The Parade, Cardiff, CF24 3AA, UK

${ }^{37}$ Steward Observatory, University of Arizona, 933 N. Cherry Avenue, Tucson, AZ 85721, USA

${ }^{38}$ Department of Astronomy \& Astrophysics, Center for Particle \& Gravitational Astrophysics, and Center for Theoretical \& Observational Cosmology, Pennsylvania State University, University Park, PA 16802, USA

${ }^{39}$ CCS Division, Los Alamos National Laboratory, Los Alamos, NM 87545, USA

${ }^{40}$ Centro de Investigaciones Energéticas, Medioambientales y Tecnológicas (CIEMAT), Madrid, Spain

${ }^{41}$ Department of Astronomy, University of California, Berkeley, 501 Campbell Hall, Berkeley, CA 94720, USA

${ }^{43}$ Lawrence Berkeley National Laboratory, 1 Cyclotron Road, Berkeley, CA 94720, USA
${ }^{42}$ Center for Cosmology and Astro-Particle Physics, The Ohio State University, Columbus, OH 43210, USA

${ }^{44}$ Department of Physics, The Ohio State University, Columbus, OH 43210, USA

${ }^{45}$ Australian Astronomical Observatory, North Ryde, NSW 2113, Australia

${ }^{46}$ George P. and Cynthia Woods Mitchell Institute for Fundamental Physics and Astronomy, and Department of Physics and Astronomy, Texas A\&M University, College Station, TX 77843, USA 


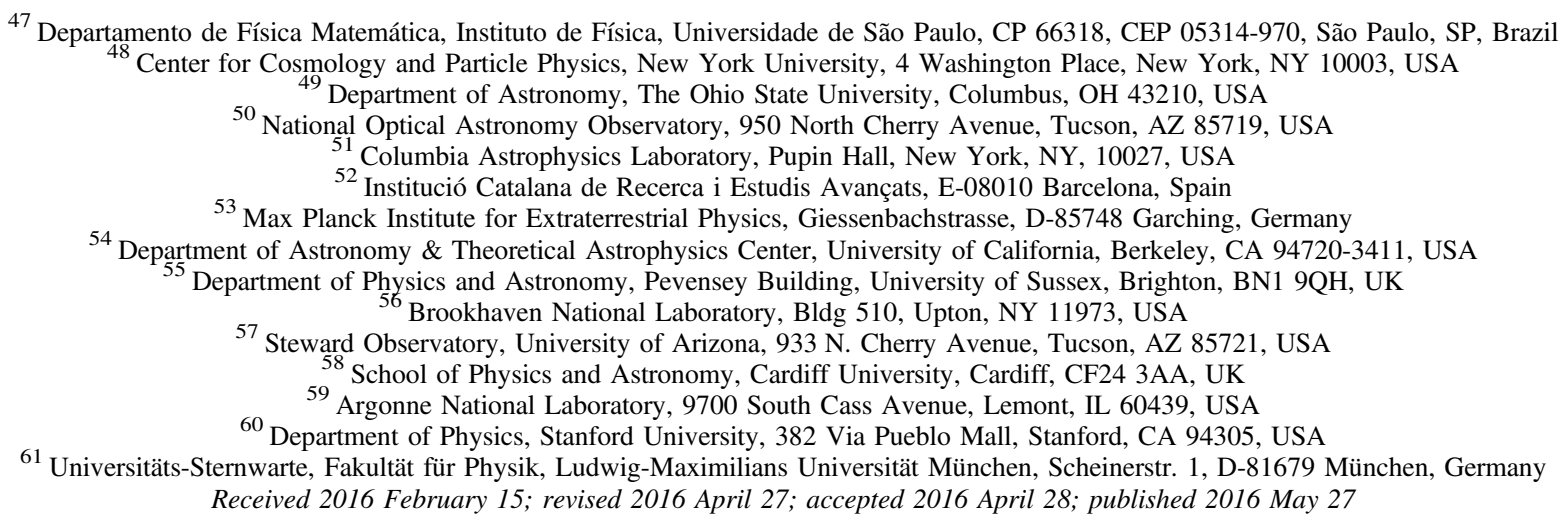

\begin{abstract}
We report the results of a deep search for an optical counterpart to the gravitational wave (GW) event GW150914, the first trigger from the Advanced LIGO GW detectors. We used the Dark Energy Camera (DECam) to image a $102 \mathrm{deg}^{2}$ area, corresponding to $38 \%$ of the initial trigger high-probability sky region and to $11 \%$ of the revised high-probability region. We observed in the $i$ and $z$ bands at 4-5, 7, and 24 days after the trigger. The median $5 \sigma$ point-source limiting magnitudes of our search images are $i=22.5$ and $z=21.8 \mathrm{mag}$. We processed the images through a difference-imaging pipeline using templates from pre-existing Dark Energy Survey data and publicly available DECam data. Due to missing template observations and other losses, our effective search area subtends $40 \mathrm{deg}^{2}$, corresponding to a $12 \%$ total probability in the initial map and 3\% in the final map. In this area, we search for objects that decline significantly between days $4-5$ and day 7 , and are undetectable by day 24 , finding none to typical magnitude limits of $i=21.5,21.1,20.1$ for object colors $(i-z)=1,0,-1$, respectively. Our search demonstrates the feasibility of a dedicated search program with DECam and bodes well for future research in this emerging field.
\end{abstract}

Key words: binaries: close - catalogs - gravitational waves - stars: neutron - surveys

\section{INTRODUCTION}

The advanced network of ground-based gravitational wave (GW) interferometers is designed to detect and study GW emission from events such as the mergers of binary systems composed of neutron stars and/or black holes to distances of hundreds of Mpc (see Abbott et al. 2016d and references therein). In mergers containing at least one neutron star, counterpart electromagnetic radiation is expected, potentially ranging from a short-duration gamma-ray burst through optical/near-IR emission from the radioactive decay of r-process nuclei to radio emission from ejecta interacting with the circumbinary medium (e.g., Li \& Paczyński 1998; Nakar \& Piran 2011; Metzger \& Berger 2012; Barnes \& Kasen 2013; Tanaka \& Hotokezaka 2013; Aasi et al. 2014; Berger 2014; Tanaka et al. 2014; Cowperthwaite \& Berger 2015). The detection of an electromagnetic counterpart will provide critical insight into the physics of the event, helping to determine the distance scale, energy scale, and the progenitor environment, as well as insight into the behavior of matter post-merger (e.g., the production of jets and outflows).

With this motivation, we recently began an observational program using the wide-field Dark Energy Camera (DECam, Flaugher et al. 2015) on the Blanco $4 \mathrm{~m}$ telescope at the Cerro Tololo Inter-American Observatory to search for optical counterparts to $\mathrm{GW}$ triggers from the new advanced $\mathrm{GW}$ detectors (LIGO, Abbott et al. 2009; Virgo, Acernese et al. 2009). This program was awarded three target of opportunity nights to observe LIGO-triggered events during the 2015B semester; observations were coordinated with and managed by the Dark Energy Survey (DES). Our program is optimized for the detection of kilonovae, the hypothesized optical counterparts of mergers involving neutron stars, which would appear as red transients with expected decay timescales of about a week (for an overview of our program see Abbott et al. 2016c, Section 5).

On 2015 September 14 at 09:50:45 UT the Advanced LIGO interferometer network detected a high-significance candidate GW event designated GW150914 (Abbott et al. 2016a) and two days later provided spatial location information in the form of probability sky maps via a private GCN circular (\#18330, Singer 2015). We initiated observations with DECam, a $3 \mathrm{deg}^{2}$ field of-view instrument, on 2015 September 18 in an effort to identify an optical counterpart. Here we describe the observations and provide the results of the three-epoch search. These DECam observations are the deepest search for an optical counterpart to GW event GW150914 (Abbott et al. 2016b).

\section{DECam OBSERVATIONS OF GW150914}

The detection of GW150914 was triggered by the coherent WaveBurst (cWB; Klimenko et al. 2008) unmodeled burst analysis during real-time data processing. On 2015 September 16, the LIGO Virgo Collaboration (LVC) provided two all-sky localization probability maps for the event, generated from the cWB and LALInferenceBurst (LIB; Veitch et al. 2015) analyses. The cWB online trigger analysis makes minimal assumptions about signal morphology by searching for coherent power across the LIGO network. The LIB analysis is a version of the LALInference analysis Bayesian forwardmodeling-based follow-up tool that uses a Sine-Gaussian signal morphology instead of models of compact binary mergers (Veitch et al. 2015); for information on both algorithms see Essick et al. (2015). The maps provided initial spatial 
Table 1

Summary of Observations

\begin{tabular}{|c|c|c|c|c|c|c|c|c|}
\hline Program & $\begin{array}{r}\text { Night } \\
\text { (UT) }\end{array}$ & MJD & $\begin{array}{c}\Delta t^{\mathrm{a}} \\
\text { (days) }\end{array}$ & $\begin{array}{c}\left\langle\mathrm{PSF}(\mathrm{FWHM})_{i}\right\rangle \\
(\operatorname{arcsec})\end{array}$ & $\langle$ airmass $\rangle$ & $\begin{array}{l}\left\langle\operatorname{depth}_{i}\right\rangle \\
(\mathrm{mag})\end{array}$ & $\begin{array}{l}\left\langle\operatorname{depth}_{z}\right\rangle \\
\text { (mag) }\end{array}$ & $\begin{array}{l}A_{\text {eff }}{ }^{\mathrm{b}} \\
\left(\mathrm{deg}^{2}\right)\end{array}$ \\
\hline \multirow[t]{2}{*}{ Main, 1st epoch } & 2015 Sep 18 & 57383 & 3.88 & 1.38 & 1.50 & 22.71 & 22.00 & 52.8 \\
\hline & 2015 Sep 19 & 57384 & 4.97 & 1.35 & 1.46 & 22.82 & 22.12 & 14.4 \\
\hline Main, 2nd epoch & 2015 Sep 21 & 57286 & 6.86 & 2.17 & 1.51 & 22.18 & 21.48 & 67.2 \\
\hline Main, 3rd epoch & 2015 Oct 08 & 57303 & 23.84 & 1.46 & 1.40 & 22.33 & 21.63 & 67.2 \\
\hline LMC, initial & 2015 Sep 18 & 57383 & 3.98 & 1.14 & 1.30 & 21.32 & 20.62 & 14.4 \\
\hline LMC, extension & 2015 Sep 27 & 57292 & 12.96 & 1.21 & 1.28 & 20.91 & 20.21 & 33.6 \\
\hline
\end{tabular}

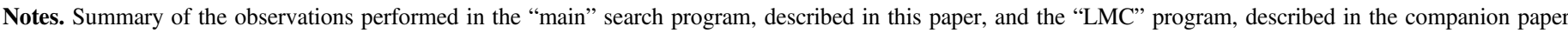

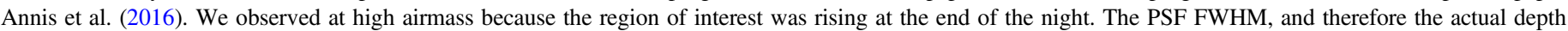

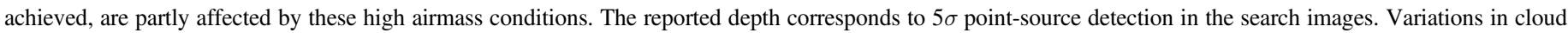

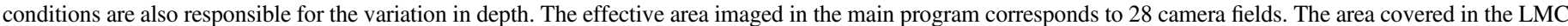
program totaled 20 fields.

a Time elapsed between the trigger time and the time stamp of the first image of the night.

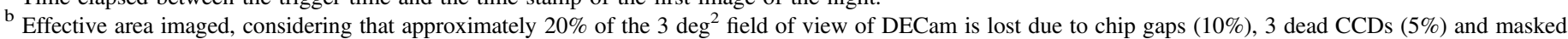
edge pixels $(5 \%)$.

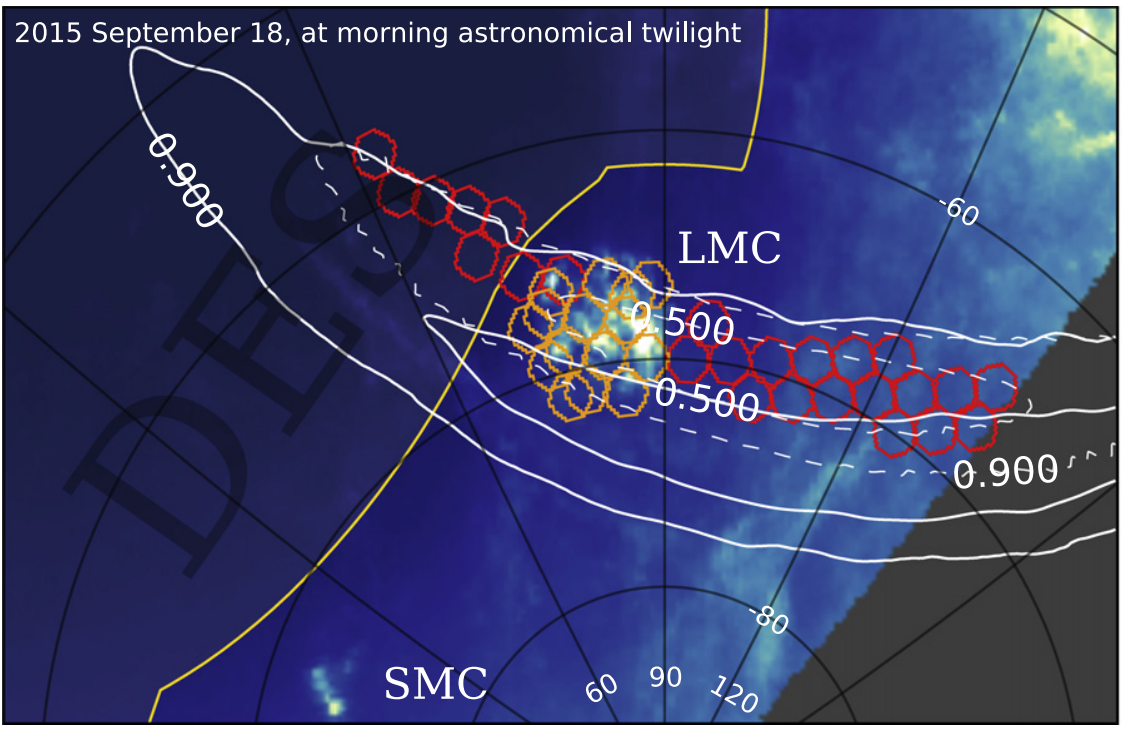

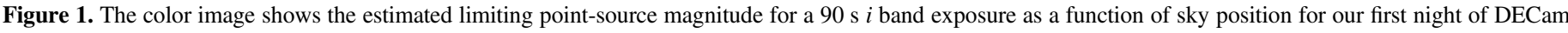

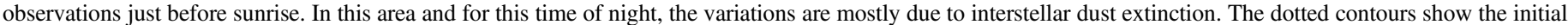

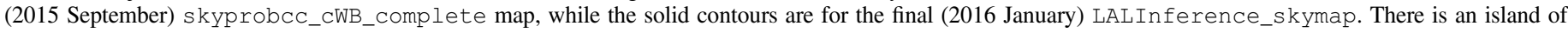

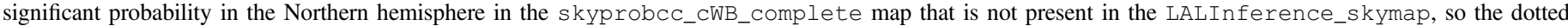

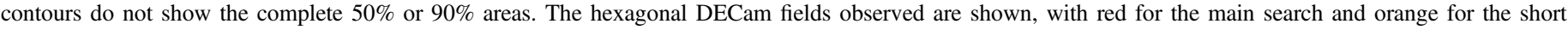

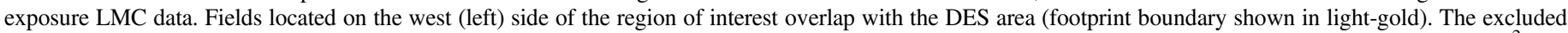

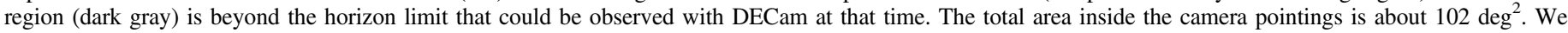

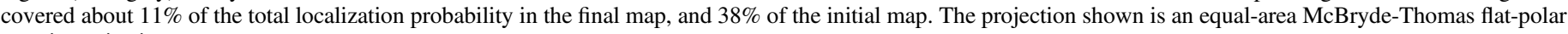
quartic projection.

localization of $50 \%$ and $90 \%$ confidence regions encompassing about 100 and $310 \mathrm{deg}^{2}$, respectively.

Our first observations with DECam took place on 2015 September 18 UT. Overall, we imaged $102 \mathrm{deg}^{2}$, covering $38 \%$ of the total probability in the initial cWB map; see Table 1 for a summary of our DECam observations. As shown in Figure 1, $18 \mathrm{deg}^{2}$ were centered on the Large Magellanic Cloud (LMC). For the remaining $84 \mathrm{deg}^{2}$ we obtained 3 separate epochs of imaging. At each epoch we acquired one $90 \mathrm{~s}$ exposure in the $i$ band and two $90 \mathrm{~s}$ exposures in the $z$ band. The first epoch spanned 4-5 days post-GW trigger (2015 September 18-19 UT), the second epoch spanned 7 days post-GW trigger (2015 September $21 \mathrm{UT}$ ), and the third was obtained 24 days postGW trigger (2015 October 08 UT).
Subsequently, in 2016 January, the LVC released a revised sky map of localization probabilities from a LALInference analysis (GCN circular \#18858, Singer 2016). That analysis used the assumption that the signal arises from a compact binary coalescence. It also showed that the data are most consistent with models of a binary black hole merger $(\mathrm{BBH})$. The LALInference-based map is considered the most accurate and authoritative localization for this event. Our $102 \mathrm{deg}^{2}$ cover a total of $11 \%$ probability in this new map, as the localization region has shifted significantly southward (see Figure 1) relative to the initial cWB map.

Our single-epoch exposures achieve median $5 \sigma$ point-source limiting magnitudes of $i=22.5$ and $z=21.8$, with an rms variation among the images of $\pm 0.5 \mathrm{mag}$. This value is a 
consequence of night-to-night variations in the observing conditions (see Table 1) and of a strong gradient in stellar density and extinction along the major axis of the region imaged (see Figure 1).

\subsection{Observing Strategy}

We chose the location and sequence of DECam observations using an automated observing strategy algorithm. The algorithm takes two inputs: the peak $i$ band absolute magnitude of the hypothesized source, and its distance. Because our program was originally designed for kilonova searches, and the $\mathrm{BBH}$ nature of the event was unknown at the time of the observations, we chose a model compatible with the kilonova models of Barnes \& Kasen (2013) and Grossman et al. (2014), with an absolute magnitude of $M_{i}=-11$ at peak, a characteristic decay time of about 1 week, and a color $i-z=1$. Since we did not have access to distance information for this event at the time, we used the nominal distance out to which LIGO was sensitive to binary neutron-star mergers: $60 \mathrm{Mpc}$. The two inputs, $M_{i}$ and distance, are used to compute an apparent magnitude for the source. Then, assuming an exposure time of $90 \mathrm{~s}$ (the nominal value used in all DES observations), we use the DES sky brightness model (Neilsen 2012), the atmospheric transmission model (using information on airmass and the interstellar dust extinction from Planck; Abergel et al. 2014), the expected seeing (from scaling laws with airmass and wavelength), and the confusion-limit probability model (based on stellar density maps) to compute, for each position in the sky, the probability that the hypothesized source would be detected by DECam. This DECam probability map is then multiplied by the LIGOprovided map to determine the region of interest for our observations. Deeper imaging is possible if called for by the model and distance.

Based on the final DECam $\times$ LIGO map, we observed the largest probability region available. The area covered had partial overlap with the DES footprint and was rising at the end of the night. The plan was to obtain 3 epochs of data covering the region of interest. Under the assumption that kilonovae are week-timescale decaying transients, we planned to take the first epoch as soon after the trigger as possible, the second epoch about two nights from the first, and the third epoch three weeks later.

In the case of GW150914 the localization region intersected the LMC. The LMC region was disfavored by our algorithm due to the high-stellar density. To explore this region, we designed a separate program that consisted of a set of short observations. We obtained $5 \mathrm{~s} i$ and $z$ band exposures covering $18 \mathrm{deg}^{2}$ centered on the LMC on 2015 September 18 and 27. This shallower data set was used to search for a potential failed supernova in the LMC; the results are reported in a separate paper (Annis et al. 2016). Figure 1 shows a sky map computed for the end of the first night of observations, zoomed in to the region of interest and detailing the fields observed in each of the three epochs in red.

Our observing strategy is suitable for binary mergers that involve at least one neutron star. In particular, the choice of $i$ and $z$ filters is driven by the peculiar colors of kilonovae. For $\mathrm{BBH}$ events, we do not expect any optical emission unless the system has a significant accretion disk, which is unlikely for stellar mass black holes. For BBH events in the upcoming
LIGO runs, we are investigating using bluer filters such as $g$ and $r$ to make our search less kilonova-specific.

\subsection{Image Processing}

Our data analysis relies on subtracting earlier template images from the science images taken for this program. In the area that overlaps the DES footprint (25\% of the total), we used DES images from the first two seasons of the survey as templates. In the $75 \%$ of the area outside of the DES footprint, we used publicly available DECam data from the NOAO Science Archive (portal-nvo.noao.edu), requiring exposures of at least $30 \mathrm{~s}$ in the $i$ and $z$ bands.

We processed the DECam search and template images using the DES Data Management single-epoch image processing software (Sevilla et al. 2011; Desai et al. 2012; Mohr et al. 2012; R. Gruendl et al. 2016, in preparation). Its output images were used as input to the difference-imaging pipeline, which we developed from the DES Supernova pipeline (Kessler et al. 2015). The main adaptation of the pipeline for our purposes was to generalize to the case of search and template images with arbitrary relative alignment. A candidate requires two SExtractor (Bertin \& Arnouts 1996) detections in the first epoch in both the $i$ and $z$ bands. To reduce the large number of detected artifacts, each detection must satisfy quality requirements (Table 3 of Kessler et al. 2015) and be selected by our automated scanning program (Goldstein et al. 2015). For each of the 2349 candidate locations, "forced" PSF-fitted fluxes and uncertainties are obtained at every epoch regardless of whether or not there was a detection.

\section{ANALYSIS}

While a BBH merger is not expected to result in an optical signature, it is nevertheless of interest to search for a possible optical counterpart. Our data was tailored for a kilonova search by choice of cadence and bandpasses. We refrained from using the key $i-z$ color cut for kilonova because the BBH nature of the merger does not call for it. We kept the assumption of a decaying transient. As our first epoch of observations occurred 4 days after the trigger, our prior on the search is that any candidate shall be fading slowly enough to be detectable 7 days after the event, but not 24 days after the event.

Of the $84 \mathrm{deg}^{2}$ area outside of the LMC, about $20 \%$ is lost due to camera fill-factor (see Table 1 for details), resulting in an effective area of $67.2 \mathrm{deg}^{2}$. In addition, $30 \%$ of the area is lost due to sparse availability of templates outside of the DES footprint. Another $10 \%$ loss arises from processing issues. This results in $40 \mathrm{deg}^{2}$, which was used in this analysis.

Based on an analysis of a sample of fake point sources injected into the images in this area, we find that the typical $80 \%$ source detection completeness in the subtracted images is at $i \approx 22.1$ and $z \approx 21.2 \mathrm{mag}$. In the first epoch, where the observing conditions were better, we achieve that level of completeness at $i \approx 22.7$ and $z \approx 21.8$, comparable to the $5 \sigma$ point-source depth for those images. The fakes were in all the images we processed, thus the completeness depth reflects the variation in conditions as well.

\subsection{Sample Selection}

For the selection criteria described below, multiple observations per night (primarily in $z$ band) are combined into a single weighted-average flux: 
Table 2

Number of Selected Events

\begin{tabular}{lrccc}
\hline \hline Mag $(i)$ & Raw & Cut 1 & Cut 2 & Cut 3 \\
\hline $18.0-18.5$ & 84 & 1 & 0 & 0 \\
$18.5-19.0$ & 177 & 1 & 0 & 0 \\
$19.0-19.5$ & 291 & 2 & 0 & 0 \\
$19.5-20.0$ & 227 & 2 & 1 & 0 \\
$20.0-20.5$ & 156 & 17 & 2 & 0 \\
$20.5-21.0$ & 225 & 42 & 3 & 0 \\
$21.0-21.5$ & 334 & 84 & 2 & 0 \\
$21.5-22.0$ & 756 & 159 & 1 & 0 \\
$22.0-22.5$ & 1099 & 183 & 0 & 0 \\
\hline Total & 2349 & 491 & 9 & 0 \\
\hline
\end{tabular}

1. Second-epoch signal-to-noise ratio $(\mathrm{S} / \mathrm{N})$ above 2 in both $i$ and $z$ (to enable flux change determination with respect to the first epoch);

2. $\geqslant 3 \sigma$ decline in both $i$ and $z$ fluxes from the first epoch to the second (to isolate fading sources; $\sigma$ is defined by the quadrature sum of the flux errors in the first two epochs);

3. $\mathrm{S} / \mathrm{N} \leqslant 3 \sigma$ in both the $i$ and $z$ third epochs (at 24 days post-trigger, to reject long-timescale transients such as supernovae).

The above criteria are consistent with typical kilonova models. Since we do not apply any selection in color, they are also consistent with other transients with timescales of about 1-2 weeks. We are not sensitive to typical timescales of GRB afterglows, as our first observation happened four days after the trigger. The cadence and bandpasses of our data did not support further generalization of the search to other classes of transients.

\subsection{Results}

In Table 2 we show the impact of our selection criteria on the sample of candidates as a function of the first epoch $i$ band magnitude. The decaying light curve requirement has the most impact in reducing the sample size. None of the candidates pass all the selection criteria. The area analyzed, $40 \mathrm{deg}^{2}$, covers $3 \%$ of the localization probability in the final LALInference map (though it covered $12 \%$ in the initial cWB map).

To interpret these results some caveats are required. Because our selection criteria impose demands on significance in the second epoch, the actual first epoch search depth depends on the decline rate and $i-z$ color of the source model. In addition, we have not yet accounted for the degraded sensitivity to candidates located in bright galaxies.

For a particular source model, we can estimate the search depth. We applied our selection criteria to a sample of fake sources randomly placed in our search images before processing with our difference-imaging pipeline. Our primary set of fakes is kilonova fakes, which we introduced using the light curves and spectra provided by Barnes \& Kasen 2013. They have a constant decay rate of $0.3 \mathrm{mag} \mathrm{day}^{-1}$ and are red, with $(i-z) \approx 1$.

The magnitude at which we recover $50 \%$ of the fakes, $m_{50 \%}$, is about 1 magnitude brighter than the $5 \sigma$ point-source limiting magnitude reported in Table 1, i.e., $m_{50 \%}-m_{5 \sigma} \approx-1$.

We did not impose color-based selection criteria but the choice of bandpasses implicitly does. Thus it is necessary to establish the performance of our analysis as a function of color, which we did by introducing fakes with similar timescales and brightness of the kilonova models, but with bluer colors. Simulations with bluer models show that for sources with $(i-z)=0$ the search depth is $m_{50 \%}-m_{5 \sigma} \approx-1.4$; for $(i-z)=-1$, it is $m_{50 \%}-m_{5 \sigma} \approx-2.4$. We therefore achieve a magnitude limit $i=21.5,21.1,20.1$ for object colors $(i-z)=1,0,-1$, respectively.

\section{CONCLUSIONS}

We presented our search for an optical counterpart to the first GW event, GW150914, using the wide-field DECam instrument. Our observations cover $102 \mathrm{deg}^{2}$ corresponding to $11 \%$ of the total probability map. The search images used in this analysis reach a median $5 \sigma$ point-source depth of $i=22.5$ and $z=21.8 \mathrm{mag}$. Our DECam/Blanco observations are the deepest optical follow-up for this GW event.

Using selection criteria that isolate fading transients over the analysis region covering $3 \%$ of the total localization probability, we find no candidate counterparts. This result is not surprising given the partial areal coverage and the BBH merger nature of the event, which is not expected to produce any optical emission. However, the work establishes a very sensitive search program, capable of detecting week-timescale transients down to magnitude limits of $i=21.5,21.1,20.1$ for source colors $(i-z)=1,0,-1$, respectively. If there is an unexpected optical counterpart associated with BBH mergers detected by LIGO, our prospects for detection in the upcoming years are good.

Prospects are also good for future events involving neutron stars: were GW150914 a binary neutron star merger, our search would have been sensitive to some kilonova models $\left(M_{i}=-15\right.$; Barnes \& Kasen 2013) out to a distance of $200 \mathrm{Mpc}$, which is about the nominal range that the GW detector network is expected to achieve in the next 3-5 years. For the more conservative model parameters that we used in the design of the observing strategy $\left(M_{i}=-11\right.$; Grossman et al. 2014), our final analysis sensitivity reaches $30 \mathrm{Mpc}$.

For the next observing campaign, we are investigating improved background rejection criteria using information such as: matching against a galaxy catalog to remove transients associated with high-redshift galaxies, angular separation between $i$ and $z$ exposures to reduce asteroids, and detailed simulations of supernovae and source models to better optimize selection requirements as well as the search strategy for future events. These improvements will allow us to perform a more sensitive search, and enable spectroscopic follow-up soon after the first epoch data is obtained.

Our search is a crucial first step and demonstrates the viability of DECam for deep optical follow-up of GW events.

Funding for the DES Projects has been provided by the U.S. Department of Energy, the U.S. National Science Foundation, the Ministry of Science and Education of Spain, the Science and Technology Facilities Council of the United Kingdom, the Higher Education Funding Council for England, the National Center for Supercomputing Applications at the University of Illinois at Urbana-Champaign, the Kavli Institute of Cosmological Physics at the University of Chicago, the Center for Cosmology and Astro-Particle Physics at the Ohio State University, the Mitchell Institute for Fundamental Physics and Astronomy at Texas A\&M University, Financiadora de Estudos e Projetos, Fundação Carlos Chagas Filho de Amparo à Pesquisa do Estado do Rio de Janeiro, Conselho Nacional de 
Desenvolvimento Científico e Tecnológico and the Ministério da Ciência, Tecnologia e Inovação, the Deutsche Forschungsgemeinschaft, and the Collaborating Institutions in the Dark Energy Survey.

The Collaborating Institutions are Argonne National Laboratory, the University of California at Santa Cruz, the University of Cambridge, Centro de Investigaciones Energéticas, Medioambientales y Tecnológicas-Madrid, the University of Chicago, University College London, the DES-Brazil Consortium, the University of Edinburgh, the Eidgenössische Technische Hochschule (ETH) Zürich, Fermi National Accelerator Laboratory, the University of Illinois at Urbana-Champaign, the Institut de Ciències de l'Espai (IEEC/CSIC), the Institut de Física d'Altes Energies, Lawrence Berkeley National Laboratory, the Ludwig-Maximilians Universität München and the associated Excellence Cluster universe, the University of Michigan, the National Optical Astronomy Observatory, the University of Nottingham, The Ohio State University, the University of Pennsylvania, the University of Portsmouth, SLAC National Accelerator Laboratory, Stanford University, the University of Sussex, and Texas A\&M University.

The DES data management system is supported by the National Science Foundation under grant No. AST-1138766. The DES participants from Spanish institutions are partially supported by MINECO under grants AYA2012-39559, ESP2013-48274, FPA2013-47986, and the Centro de Excelencia Severo Ochoa SEV-2012-0234. Research leading to these results has received funding from the European Research Council under the European Unions Seventh Framework Programme (FP7/2007-2013), including ERC grant agreements 240672, 291329, and 306478.

This research uses services or data provided by the NOAO Science Archive. NOAO is operated by the Association of Universities for Research in Astronomy (AURA), Inc. under a cooperative agreement with the National Science Foundation.

\section{REFERENCES}

Aasi, J., Abadie, J., Abbott, B. P., et al. 2014, ApJS, 211, 7

Abbott, B. P., Abbott, R., Abbott, T. D., et al. 2016a, PhRvL, 116, 061102

Abbott, B. P., Abbott, R., Abbott, T. D., et al. 2016b, ApJL, in press (arXiv:1602.08492)

Abbott, B. P., Abbott, R., Abbott, T. D., et al. 2016d, LRR, 19, 1

Abbott, B. P., Abbott, R., Adhikari, R., et al. 2009, PhRvD, 80, 102001

Abbott, T., Abdalla, F. B., Allam, S., et al. 2016c, MNRAS, in press (arXiv:1601.00329)

Abergel, A., Ade, P. A. R., Aghanim, N., et al. 2014, A\&A, 571, A11

Acernese, F., Alshourbagy, M., Antonucci, F., et al. 2009, CQGra, 26, 085009

Annis, J., Soares-Santos, M., Berger, E., et al. 2016, ApJL, in press (arXiv:1602.04199)

Barnes, J., \& Kasen, D. 2013, ApJ, 775, 18

Berger, E. 2014, ARA\&A, 52, 43

Bertin, E., \& Arnouts, S. 1996, A\&AS, 117, 393

Cowperthwaite, P. S., \& Berger, E. 2015, ApJ, 814, 25

Desai, S., Armstrong, R., Mohr, J. J., et al. 2012, ApJ, 757, 83

Essick, R., Vitale, S., Katsavounidis, E., Vedovato, G., \& Klimenko, S. 2015, ApJ, 800, 81

Flaugher, B., Diehl, H. T., Honscheid, K., et al. 2015, AJ, 150, 150

Goldstein, D. A., D’Andrea, C. B., Fischer, J. A., et al. 2015, AJ, 150, 82

Grossman, D., Korobkin, O., Rosswog, S., \& Piran, T. 2014, MNRAS, 439, 757

Kessler, R., Marriner, J., Childress, M., et al. 2015, AJ, 150, 172

Klimenko, S., Yakushin, I., Mercer, A., \& Mitselmakher, G. 2008, CQGra, 25, 114029

Li, L.-X., \& Paczyński, B. 1998, ApJL, 507, L59

Metzger, B. D., \& Berger, E. 2012, ApJ, 746, 48

Mohr, J. J., Armstrong, R., Bertin, E., et al. 2012, Proc. SPIE, 8451, $84510 \mathrm{D}$

Nakar, E., \& Piran, T. 2011, Natur, 478, 82

Neilsen, E. H., Jr. 2012, in ASP Conf. Ser. 461, Astronomical Data Analysis Software and Systems XXI, ed. P. Ballester, D. Egret, \& N. P. F. Lorente (San Francisco, CA: ASP), 201

Sevilla, I., Armstrong, R., Bertin, E., et al. 2011, arXiv:1109.6741

Singer, L. 2015, GCN, 18330, 1

Singer, L. 2016, GCN, 18858, 1

Tanaka, M., \& Hotokezaka, K. 2013, ApJ, 775, 113

Tanaka, M., Hotokezaka, K., Kyutoku, K., et al. 2014, ApJ, 780, 31

Veitch, J., Raymond, V., Farr, B., et al. 2015, PhRvD, 91, 042003 